\title{
LINEAR MAPS PRESERVING THE MINIMUM MODULUS
}

\author{
Abdellatif Bourhim AND María Burgos
}

\begin{abstract}
We characterize surjective linear maps that preserve the minimum modulus between unital semisimple Banach algebras, one of them is a unital $C^{*}$-algebra having either real rank zero or essential socle. We also describe surjective linear maps on $\mathscr{L}(H)$, with $H$ an infinitedimensional Hilbert space, preserving the essential minimum modulus. Results concerning surjectivity and maximum modulus are also obtained.
\end{abstract}

Mathematics subject classification (2010): Primary 47B49; Secondary 47A10, 47D25. modulus.

Keywords and phrases: $C^{*}$-algebra, $\mathrm{JB}^{*}$-algebra, real rank zero, socle, minimum modulus, surjectivity

\section{REFERENCES}

[1] P. AiEnA, Fredholm and local spectral theory, with applications to Multipliers, Kluwer Academic Publishers, 2004.

[2] B. Aupetit, Primer on spectral theory, Springer-Verlag, New York, 1991.

[3] B. Aupetit, AND H. T. Mouton, Spectrum preserving linear mappings in Banach algebras, Studia Math., 109, 1 (1994), 91-100.

[4] B. AuPETIT, Spectrum-preserving linear map between Banach algebra or Jordan-Banach algebra, J. London Math. Soc., 62 (2000), 917-924.

[5] R. Bouldin, The essential minimum modulus, Indiana Univ. Math. J., 30 (1981), 513-517.

[6] M. BREŠAR, A. FoŠNER AND P. ŠEMRL, A note on invertibility preservers on Banach algebras, Proc. Amer. Math. Soc., 131 (2003), 3833-3837.

[7] M. BREŠAR AND P. ŠEMRL, Linear maps preserving the spectral radius, J. Funct. Anal., 142, 2 (1996), 360-368.

[8] L. G. Brown And G. K. Pedersen, $C^{*}$-algebras of rank real zero, J. Funct. Anal., 99 (1991), $131-149$.

[9] P. R. CHERnoff, Representations, automorphisms and derivations of some operator algebras, J. Funct. Anal., 12 (1973), 275-289.

[10] K. R. Davidson, $C^{*}$-Algebras by Example, Fields Institute Monographs, vol. 6, American Mathematical Society, Rhode Island, 1996.

[11] I. N. HeRsteIn, Jordan homomorphisms, Trans. Amer. Math. Soc., 81 (1956), 331-341.

[12] A. Kaidi, A. Morales, AND A. Rodríguez, A holomorphic characterization of $C^{*}$ - and $J B^{*}$ algebras, Manuscripta Math., 104 (2001), 467-478.

[13] R. V. KADISON, Isometries of operator algebras, Ann. Math., 54 (1951), 325-338.

[14] M. Mathieu, Elementary operators on prime $C^{*}$-algebras II., Glasgow Math. J., 30 (1988), 275-284.

[15] M. Mathieu, Spectrally bounded operators on simple $C^{*}$-algebras, Proc. Amer.Math. Soc., 132 (2004), 443-446.

[16] M. MвекHTA, Linear maps preserving the minimum and surjectivity moduli of operators, preprint.

[17] V. MÜLLER, Spectral theory of linear operators and spectral systems in Banach algebras, Operator Theory: Advances and Applications, 139. Birkhäuser Verlag, Basel, 2003.

[18] J. PÉREZ, L. RICO, AND A. RodRíGUEZ, Full subalgebras of Jordan-Banach algebras and algebra norms on JB* -algebras, Proc. Amer. Math. Soc., 121 (1994), 1133-1143.

[19] A. RodRÍGueZ, Jordan axioms for $C^{*}$-algebras, Manuscripta Math., 61 (1988), 297-314.

[20] B. Russo AND H. A. Dye, A note on unitary operators in $C^{*}$-algebras, Duke Math. J., 33 (1966), 413-416. 
[21] R. D. Schaefer, An introduction to nonassociative algebras, Academic Press, New York, 1966.

[22] J.D.M. WRIGHT, Jordan $C^{*}$-algebras, Michigan Math. J., 24 (1977), 291-302.

[23] M. A. Youngson, Non unital Banach Jordan algebras and $C^{*}$-triple systems, Proc. Edinburgh Math. Soc., 24 (1981), 19-31.

[24] J. ZEMÁNEK, Geometric interpretations of the essential minimum modulus in Invariant subspaces and other topics, Oper. Theory, Adv. Appl., vol. 6, Birkhäuser Verlag, Basel, 1982. 